\title{
Automatic reactivity characterisation of char particles from pulverised coal combustion using computer vision
}

\author{
Deisy Chaves*,+ \\ * School of Systems Engineering and Computing, Universidad del Valle, Cali, Colombia \\ + Dept. of Electrical, Systems and Automatic Engineering, Universidad de León, León, Spain \\ Advisors: Maria Trujillo*, Enrique Alegre ${ }^{+}$and Laura Fernández-Robles ${ }^{+}$ \\ Received 30 September 2019; revised 17 December 2019; accepted 9 January 2020
}

\begin{abstract}
Char morphologies produced during pulverised coal combustion may determine coal reactivity which affects the combustion efficiency and the emissions of $\mathrm{CO}_{2}$ in power plants. Commonly, char samples are characterised manually, but this process is subjective and time-consuming. This work proposes methods to automate the char reactivity characterisation using microscopy images and computer vision techniques. These methods are summarised in three contributions: the localisation of char particles based on candidate regions and deep learning methods; the classification of particles into char reactivity groups using morphological and texture features; and the integration in a system of the two previous proposals to characterise char sample reactivity. The proposed system successfully estimate char reactivity in a fast and accurate way.
\end{abstract}

\section{Introduction}

Pulverised coal combustion is a technology extensively used in power plants for generating electricity. Char particles with distinctive morphologies are generated in the first stage of the combustion. These morphologies may be used to estimate coal reactivity which determine the performance of the combustion and air emissions [1]. Typically, a char sample is characterised by an expert who observes up to 500 particles in a char-block surface through a microscope. This process is subjective, error-prone and time-consuming. The automatic char sample characterisation is still an open problem in computer vision due to several factors that difficult the correct detection and classification of chars, such as broken walls due to the char generation process, blurriness and scratches caused by poor char-block polishing [1]. This study ${ }^{\dagger}$ proposes several methods to automatically characterise char reactivity using microscopy images from various coal samples and computer vision techniques.

\section{Methods and results}

This research presents contributions in three research lines: the localisation of char particles, the classification of chars into reactivity groups, and the integration in a system of both methods to estimate char reactivity.

Correspondence to: <dchas@unileon.es, deisy.chaves@correounivalle.edu.co>

Recommended for acceptance by Dena Bazazian and Xim Cerda https://doi.org/10.5565/rev/elcvia.1191

ELCVIA ISSN:1577-5097

Published by Computer Vision Center / Universitat Autònoma de Barcelona, Barcelona, Spain

† https://buleria.unileon.es/handle/10612/9588 
The localisation of char particles is addressed by two methods. The first method uses texture descriptors and a Support Vector Machine classifier (SVM) to detect char particles by determining if a candidate region contains a char particle or not. Candidate regions are generated by combining regions and edge information. This method achieved better detection results - a recall of 0.58 and a precision of 0.72 - in comparison to the ones obtained using Edge Boxes [2]. The second method uses deep learning for the detection of four object categories contained in images: char particles $(\mathrm{CH})$, unburned coal particles (UB), undefined particles (UD) due to uneven char-block surfaces, and artifacts (AF) due to poor char-block polishing. Objects of interest are detected using enhanced images based on closing operations and a combination of the results obtained by Faster-RCNN, a deep-learning-based object detector. This method achieved Average Precision-Recall values of 0.809 for $\mathrm{CH}, 0.399$ for $\mathrm{UB}, 0.372 \mathrm{UD}$, and 0.478 for $\mathrm{AF}$. The use of enhancement techniques to improve the appearance of input images outperformed the results obtained directly with the original images.

The classification of char particles, from coal samples of three Colombian regions, is conducted using supervised learning and three methods are proposed. Two of the proposed methods are focused on classifying particles into two char reactivity groups: high and low reactive. These methods use global morphological features and the SIFT features with Bag-of-Word representation, and are compared to the decision tree proposed by the International Committee for Coal and Organic Petrology (ICCP) [4]. The classification results showed that a combination of global morphological features - SVM accuracy (Acc.) of 99.86- improved the results observed with the ICCP decision tree and SIFT features - SVM Acc. of 93.03- [5, 3]. The third method evaluates a concatenation of global and local features to classify char particles into high, medium, or low char reactive groups. Features are obtained from grey level images and bit-slice images. An improvement of the classification accuracy was observed using bit-slice images and feature concatenation - SVM Acc. of 93.98in comparison to the results yielded using transfer learning on CNNs -Acc. of 86.34- [6].

Finally, a system to estimate char sample reactivity is proposed by integrating char particles detection and classification methods. The generalisation capability of the system was evaluated using char images from different coal samples. In most of the cases, the system allowed to successfully classify char particles into reactivity groups and characterise char sample reactivity correctly even when the system was trained and test using char images acquired from coals of different regions.

\section{Conclusion}

This work provided successful solutions to automatically characterise char reactivity in microscopy images of char-blocks from different coal samples using computer vision techniques.

\section{References}

[1] Wu T., Lester E. and Cloke M., "Advanced Automated Char Image Analysis Techniques", Energy \& Fuels 20(3):1211-1219, 2006.

[2] Chaves D., Trucco E., Barraza J., and Trujillo M., "An image processing system for char reactivity combustion", Computer in Industry 106:60-70, 2019.

[3] Chaves D., Trujillo M., Garcia Ed., Barraza J., Lester Ed., Barajas M., Rodriguez B., Romero M., and Fernndez-Robles L., "Automated inspection of char morphologies in colombian coals using image analysis", Intelligent Automation and Soft Computing 1-9, 2019.

[4] Alvarez D. and Lester E. "Atlas of Char Occurrences. Commission III Combustion Working Group", Internacional Conference on Coal Petrology, 2001.

[5] Chaves D., Trujillo M., and Barraza J.M., "Global and local features for char image classification", IWINAC, Elche - Spain, 9107:99-107, 2015.

[6] Chaves D., Fernndez-Robles L., Bernal J., Alegre E., and Trujillo M., "Automatic characterisation of chars from the combustion of pulverised coals using machine vision", Powder Technology 338:110-118, 2018. 